\title{
Phase space localization of Riesz bases for $L^{2}\left(\mathbb{R}^{d}\right)$
}

\author{
Karlheinz Gröchenig and Eugenia Malinnikova
}

\begin{abstract}
We prove a strong uncertainty principle for Riesz bases in $L^{2}\left(\mathbb{R}^{d}\right)$ and show that the orthonormal basis constructed by Bourgain possesses the optimal phase space localization.
\end{abstract}

\section{Introduction}

In [7] J. Bourgain constructed an orthonormal basis for $L^{2}(\mathbb{R})$ consisting of functions $f_{n} \in L^{2}(\mathbb{R})$, such that

$$
\sup _{n \in \mathbb{N}}\left(\inf _{a \in \mathbb{R}} \int_{\mathbb{R}}|x-a|^{2}\left|f_{n}(x)\right|^{2} d x+\inf _{b \in \mathbb{R}} \int_{\mathbb{R}}|\xi-b|^{2}\left|\widehat{f_{n}}(\xi)\right|^{2} d \xi\right)<\infty .
$$

Bourgain remarked that the exponent 2 of $|x-a|$ and $|\xi-b|$ is optimal and that there are no orthonormal bases with a better phase space localization.

In this paper we prove the following strong uncertainty principle for Riesz bases for $L^{2}\left(\mathbb{R}^{d}\right)$ :

Theorem 1.1. If $\left\{f_{n}\right\}_{n=1}^{\infty}$ is a Riesz basis for $L^{2}\left(\mathbb{R}^{d}\right)$ and $s>d$, then

$$
\sup _{n \in \mathbb{N}}\left(\inf _{a \in \mathbb{R}^{d}} \int_{\mathbb{R}^{d}}|x-a|^{2 s}\left|f_{n}(x)\right|^{2} d x+\inf _{b \in \mathbb{R}^{d}} \int_{\mathbb{R}^{d}}|\xi-b|^{2 s}\left|\widehat{f_{n}}(\xi)\right|^{2} d \xi\right)=\infty .
$$

This theorem therefore asserts that the Bourgain basis possesses the best possible phase space localization. For the case of an orthonormal basis for $L^{2}(\mathbb{R})$ in dimension $d=1$, Bourgain outlines a proof strategy for Theorem 1.1. Precisely, he writes that "it has been shown by $T$. Steger that $L^{2}(\mathbb{R})$ does not admit a basis of the form $f_{j}=e^{i b_{j} x} g_{j}\left(x-a_{j}\right)$, where $g_{j}$ satisfies $\sup _{j}\left\|g_{j}\right\|_{A_{\epsilon}}<\infty$, defining

$$
\|g\|_{A_{\epsilon}}^{2}=\int\left(1+x^{2}\right)^{1+\epsilon}|g(x)|^{2} d x+\int\left(1+\xi^{2}\right)^{1+\epsilon}|\widehat{g}(\xi)|^{2} d \xi .
$$

Here $\epsilon>0$ is any strictly positive number. His argument is based on the fact that the operations $x$ ( $x$-multiplication) and $d / d x$ in the latter basis would become 'almost' diagonal operators, violating the non-commutation property $[d / d x, x]=I$.

Mathematics Subject Classification (2010): Primary 81P99, 81S05; Secondary 42C15. Keywords: Uncertainty principle, Balian-Low theorem, Riesz basis, localized frame. 
He also makes use of a density computation due to $Y$. Meyer of the set $\Lambda$ of pairs $\left(a_{j}, b_{j}\right)$ in phase space. The condition $\epsilon>0$ is important in Steger's argument as well as for Meyer's distribution result to be valid"; see [7].

Some of these arguments have made their way into the literature. A density argument related to Meyer's argument has appeared in the fundamental paper of Ramanathan and Steger [23] on the density of Gabor frames and has become the main technique for investigating the density of frames. See [2], [16], and [17] for some variations of the Ramanathan-Steger technique. The canonical commutation relations were used in Battle's elegant proof of the Balian-Low theorem [3].

However, a full proof of the uncertainty principle of Theorem 1.1 has not yet been given. Research has focused mainly on bases consisting of phase space shifts $f_{n}(x)=e^{2 \pi i b_{n} x} g\left(x-a_{n}\right)$ of a single generating function $g$, the so-called Gabor systems. The theorem of Balian-Low asserts that a basis satisfying (1.1) cannot consist of a (regular) Gabor system. We refer the reader to proofs of the theorem in [10] and [3], to the survey articles on the Balian-Low theorem and its generalizations ([5] and [9]), and to the monograph [14] for detailed discussions of the subject. Gabor systems are somewhat easier to handle, because one needs to control the localization of only one function in contrast to Bourgain's case.

In this paper we offer a complete proof of Theorem 1.1 which extends the result mentioned in [7] to higher dimensions and to Riesz bases instead of orthonormal bases. For orthogonal bases our proof follows the outline of Bourgain. The case of Riesz bases requires additional ideas. We will apply the theory of localized frames [12], [15] to verify that the biorthogonal basis possesses the same localization properties as the original basis. In a second step we use a bootstrap argument. We will show that if a Riesz basis violates condition (1.2) for $s>d$, then we can construct a new Riesz basis with optimal phase space localization, for instance, with all functions in a Gelfand-Shilov space of test functions.

It may seem a lot of effort to prove the non-existence of well localized phase space bases, but several arguments are of interest in themselves. The proof combines tools from the density theory of frames, the canonical commutation relations, the theory of localized frames, recent phase space methods, and a new argument of how to improve the quality of a given basis.

One of the corollaries of Theorem 1.1 is that there is no Riesz basis of phase space shifts of the Gaussian function in $L^{2}\left(\mathbb{R}^{d}\right)$. This fact implies that there is no subset $\Lambda \subset \mathbb{C}^{d}$ that is both sampling and interpolating for the Bargmann-Fock space $\mathcal{F}^{2}\left(\mathbb{C}^{d}\right)$. This statement is well known in dimension $d=1$, but seems to have been open in higher dimensions.

The paper is organized as follows: in Section 2 we show that a well-localized phase space basis must be indexed by a set of density one. In Section 3 we prove Theorem 1.1 for the special case when its biorthogonal basis is also well localized; this includes the case of an orthonormal basis. Section 4 contains some background about time-frequency analysis. In Section 5 we develop the necessary arguments to prove the uncertainty principle of Theorem 1.1 for Riesz bases. Section 6 elaborates the non-existence of sets of simultaneous sampling and interpolation and concludes with further remarks. 


\section{Density conditions}

We say that a sequence of functions $\left\{f_{n}\right\}_{n=1}^{\infty} \subset L^{2}\left(\mathbb{R}^{d}\right)$ has phase space localization of magnitude $s$, if

$$
\sup _{n \in \mathbb{N}}\left(\inf _{a \in \mathbb{R}^{d}} \int_{\mathbb{R}^{d}}|x-a|^{2 s}\left|f_{n}(x)\right|^{2} d x+\inf _{b \in \mathbb{R}^{d}} \int_{\mathbb{R}^{d}}|\xi-b|^{2 s}\left|\widehat{f_{n}}(\xi)\right|^{2} d \xi\right)<\infty .
$$

In this case there exist points $\left(a_{n}, b_{n}\right) \in \mathbb{R}^{2 d}$, such that

$$
\sup _{n \in \mathbb{N}}\left(\int_{\mathbb{R}^{d}}\left|x-a_{n}\right|^{2 s}\left|f_{n}(x)\right|^{2} d x+\int_{\mathbb{R}^{d}}\left|\xi-b_{n}\right|^{2 s}\left|\widehat{f_{n}}(\xi)\right|^{2} d \xi\right)<\infty .
$$

Then the set $\Lambda=\left\{\left(a_{n}, b_{n}\right)\right\}_{n=1}^{\infty}$ is the set in the phase space where the functions $\left\{f_{n}\right\}_{n}$ are localized. Note that there is some freedom in the choice of points $\left(a_{n}, b_{n}\right) \in \mathbb{R}^{2 d}$.

We will first estimate the density of the set $\Lambda=\left\{\left(a_{n}, b_{n}\right)\right\}_{n=1}^{\infty} \subset \mathbb{R}^{2 d}$ both for Riesz bases and frames for $L^{2}\left(\mathbb{R}^{d}\right)$ which have phase space localization. The ideas we follow are well known, see [23], [24], [21], and [25].

Let $\Lambda$ be a subset of $\mathbb{R}^{2 d}$. We denote by $D^{+}(\Lambda)$ and $D^{-}(\Lambda)$ its upper and lower Beurling densities,

$$
\begin{aligned}
& D^{+}(\Lambda)=\limsup _{r \rightarrow \infty} \sup _{x \in \mathbb{R}^{2 d}} \frac{\operatorname{card}(\Lambda \cap Q(x, r))}{|Q(x, r)|}, \\
& D^{-}(\Lambda)=\liminf _{r \rightarrow \infty} \inf _{x \in \mathbb{R}^{2 d}} \frac{\operatorname{card}(\Lambda \cap Q(x, r))}{|Q(x, r)|},
\end{aligned}
$$

where $x=\left(x_{1}, x_{2}\right) \in \mathbb{R}^{d} \times \mathbb{R}^{d}$ and

$$
Q(x, r)=\left\{\left(y_{1}, y_{2}\right) \in \mathbb{R}^{d} \times \mathbb{R}^{d}:\left|x_{1}-y_{1}\right|<r,\left|x_{2}-y_{2}\right|<r\right\} .
$$

These densities can be also defined by using dilations of cubes or balls in $\mathbb{R}^{2 d}$ instead of $Q(x, r)$, as was proved by Landau [22].

A set $\Lambda \subset \mathbb{R}^{2 d}$ is relatively separated if $\sup _{x \in \mathbb{R}^{2 d}} \operatorname{card}\left(\Lambda \cap\left(x+[0,1]^{2 d}\right)\right)<\infty$. Clearly, if $D^{+}(\Lambda)<\infty$, then $\Lambda$ is relatively separated.

Lemma 2.1. Suppose that $\left\{f_{n}\right\}_{n=1}^{\infty}$ is a Riesz basis for $L^{2}\left(\mathbb{R}^{d}\right)$ that has phase space localization of magnitude $s, s>0$, at points $\left\{\left(a_{n}, b_{n}\right)\right\}_{n=1}^{\infty}$, i.e.,

$$
\sup _{n \in \mathbb{N}}\left(\int_{\mathbb{R}^{d}}\left|x-a_{n}\right|^{2 s}\left|f_{n}(x)\right|^{2} d x+\int_{\mathbb{R}^{d}}\left|\xi-b_{n}\right|^{2 s}\left|\widehat{f_{n}}(\xi)\right|^{2} d \xi\right)=S<\infty .
$$

Then $\Lambda=\left\{\left(a_{n}, b_{n}\right)\right\}_{n=1}^{\infty} \subset \mathbb{R}^{2 d}$ is relatively separated and $D^{+}(\Lambda) \leq 1$.

Proof. Fix $\epsilon>0$. We say that a function $g \in L^{2}\left(\mathbb{R}^{d}\right)$ is $\epsilon$-concentrated on some set $E \subset \mathbb{R}^{d}$ if

$$
\int_{E}|g(x)|^{2} d x \geq\left(1-\epsilon^{2}\right)\|g\|^{2} .
$$


Since

$$
\int_{\left|x-a_{n}\right| \geq r}\left|f_{n}(x)\right|^{2} d x \leq r^{-2 s} \int_{\left|x-a_{n}\right| \geq r}\left|x-a_{n}\right|^{2 s}\left|f_{n}(x)\right|^{2} d x \leq r^{-2 s} S,
$$

there exists $r=r(\epsilon)$ such that $f_{n}$ is $\epsilon$-concentrated on $B\left(a_{n}, r\right)$ uniformly in $n$. Likewise $\widehat{f_{n}}$ is $\epsilon$-concentrated on $B\left(b_{n}, r\right)$ for every $n$. We fix $\left(x_{0}, \xi_{0}\right) \in \mathbb{R}^{d} \times \mathbb{R}^{d}$, consider any $R>0$ and denote $Q_{R}=Q\left(\left(x_{0}, \xi_{0}\right), R\right)$. Note that if $\left(a_{n}, b_{n}\right) \in Q_{R}$, then $f_{n}$ is $\epsilon$-concentrated on $B\left(x_{0}, R+r\right)$, and $\widehat{f_{n}}$ is $\epsilon$-concentrated on $B\left(\xi_{0}, R+r\right)$, where $r=r(\epsilon)$ as above.

We now apply a standard estimate of the trace of a time-frequency restriction operator to conclude that $D^{+}(\Lambda) \leq 1$, see [23].

Let $\mathcal{F}$ be the Fourier transform and $P_{E}$ be the projection operator $P_{E} f=$ $\chi_{E} f$ (multiplication of $f$ by the characteristic function of $E$ ). The phase space restriction operator is defined by

$$
L=P_{B\left(x_{0}, R+r\right)}\left(\mathcal{F}^{-1} P_{B\left(\xi_{0}, R+r\right)} \mathcal{F}\right) P_{B\left(x_{0}, R+r\right)}=P_{1} P_{2} P_{1} .
$$

It is well known, see for example [11], that

$$
\operatorname{tr}(L)=\left|B\left(x_{0}, R+r\right)\right|\left|B\left(\xi_{0}, R+r\right)\right|=\left|Q_{R+r}\right| .
$$

For each $f_{n}$ such that $\left(a_{n}, b_{n}\right) \in Q_{R}$, we have

$$
\left\|f_{n}-L f_{n}\right\| \leq\left\|f_{n}-P_{1} f_{n}\right\|+\left\|P_{1}\right\|\left\|f_{n}-P_{2} f_{n}\right\|+\left\|P_{1} P_{2}\right\|\left\|f_{n}-P_{1} f_{n}\right\| \leq 3 \epsilon\left\|f_{n}\right\| .
$$

Now let $\left\{g_{n}\right\}$ be the biorthogonal basis for $\left\{f_{n}\right\}$, i.e., $\left(f_{n}, g_{m}\right)=\delta_{n m}$. Then

$$
\begin{aligned}
\operatorname{tr}(L) & \geq \sum_{\left(a_{n}, b_{n}\right) \in Q_{R}}\left(L f_{n}, g_{n}\right) \geq \sum_{\left(a_{n}, b_{n}\right) \in Q_{R}}\left(\left(f_{n}, g_{n}\right)-\left|\left(f_{n}-L f_{n}, g_{n}\right)\right|\right) \\
& \geq(1-3 C \epsilon) \operatorname{card}\left(\Lambda \cap Q_{R}\right),
\end{aligned}
$$

where $C=\sup _{n}\left\|f_{n}\right\|\left\|g_{n}\right\|<\infty$ (since $\left\{f_{n}\right\}$ is a Riesz basis). Thus

$$
\operatorname{card}\left(\Lambda \cap Q\left(\left(x_{0}, \xi_{0}\right), R\right)\right) \leq(1-3 C \epsilon)^{-1}\left|Q\left(\left(x_{0}, \xi_{0}\right), R+r\right)\right|
$$

Taking the limit $R \rightarrow \infty$, we obtain $D^{+}(\Lambda) \leq(1-3 C \epsilon)^{-1}$ for every $\epsilon>0$, and thus $D^{+}(\Lambda) \leq 1$, and $\Lambda$ is relatively separated.

Remark. Lemma 2.1 and its proof hold also for Riesz sequences. If $\left\{f_{n}\right\}_{n=1}^{\infty}$ is a frame that has phase space localization of magnitude $s>0$ at points $\left\{\left(a_{n}, b_{n}\right)\right\}_{n=1}^{\infty}$ and satisfies $C^{-1} \leq\left\|f_{n}\right\|_{2} \leq C$, then it is still true that $\Lambda=\left\{\left(a_{n}, b_{n}\right)\right\}_{n=1}^{\infty}$ is a relatively separated set and that $D^{+}(\Lambda)<\infty$. This follows by compactness arguments, see Theorem 3.5 in [19] for a similar result in dimension $d=1$.

Lemma 2.2. Suppose that $\left\{f_{n}\right\}_{n=1}^{\infty}$ is a frame for $L^{2}\left(\mathbb{R}^{d}\right)$ with $C^{-1} \leq\left\|f_{n}\right\|_{2} \leq$ $C<\infty$. If $s>d$ and $\left\{f_{n}\right\}_{n}$ has phase space localization of magnitude $s$ at points $\left\{\left(a_{n}, b_{n}\right)\right\}_{n}$, then $\Lambda=\left\{\left(a_{n}, b_{n}\right)\right\}_{n=1}^{\infty}$ is relatively separated and $D^{-}(\Lambda) \geq 1$. 
We remark that the lemma does not hold for $s=d$. This can be seen from the construction of an orthonormal basis in [7].

Proof. Let $K(y, l)$ denote the cube with center $y \in \mathbb{R}^{q}$ and side length $2 l$,

$$
K(y, l)=\left\{z \in \mathbb{R}^{q}:\|y-z\|_{\infty}<l\right\},
$$

where $\|z\|_{\infty}=\max _{1 \leq s \leq q}\left|z_{s}\right|, z=\left(z_{1}, \ldots, z_{q}\right) \in \mathbb{R}^{q}$.

Fix $\epsilon>0$ and choose $\delta$ in the open interval $(d / s, 1)$. This is possible by the hypothesis $s>d$.

Step 1. An estimate for the coefficients $\left(\psi, f_{m}\right)$ of a localized function. Assume that $\psi \in L^{2}\left(\mathbb{R}^{d}\right),\|\psi\|_{2}=1, \psi$ is $\epsilon$-concentrated on $K\left(a, R-R^{\delta}\right)$, and its Fourier transform is supported on $K\left(b, R-R^{\delta}\right)$. Set $\eta=\psi\left(1-\chi_{K\left(a, R-R^{\delta}\right)}\right)$, so that $\|\eta\|_{2} \leq \epsilon$.

If $\left\|a_{m}-a\right\|_{\infty}>2^{k} R$, then the following estimate holds:

$$
\begin{aligned}
& \left|\left(\psi, f_{m}\right)\right|^{2} \leq 2\left|\left(\psi\left(1-\chi_{K\left(a, R-R^{\delta}\right)}\right), f_{m}\right)\right|^{2}+2\left(\int_{K\left(a, R-R^{\delta}\right)}|\psi(x)|\left|f_{m}(x)\right| d x\right)^{2} \\
& \quad \leq 2\left|\left(\eta, f_{m}\right)\right|^{2}+2\left(\left(\left(2^{k}-1\right) R+R^{\delta}\right)^{-s} \int_{K\left(a, R-R^{\delta}\right)}\left|x-a_{m}\right|^{s}|\psi(x)|\left|f_{m}(x)\right| d x\right)^{2} \\
& \quad \leq 2\left|\left(\eta, f_{m}\right)\right|^{2}+2\left(\left(2^{k}-1\right) R+R^{\delta}\right)^{-2 s} S .
\end{aligned}
$$

If $\left\|b_{m}-b\right\|_{\infty}>2^{k} R$, then

$$
\left|\left(\psi, f_{m}\right)\right|^{2}=\left|\left(\widehat{\psi}, \widehat{f_{m}}\right)\right|^{2}=\left(\int_{K\left(b, R-R^{\delta}\right)}|\widehat{\psi}(\xi)|\left|\widehat{f_{n}}(\xi)\right| d \xi\right)^{2} \leq\left(\left(2^{k}-1\right) R+R^{\delta}\right)^{-2 s} S .
$$

Let $\mathcal{M}_{0}=\left\{n:\left(a_{n}, b_{n}\right) \in K(a, R) \times K(b, R)\right\}$ and let $\mathcal{M}$ be the complement of $\mathcal{M}_{0}$, $\mathcal{M}=\left\{n:\left(a_{n}, b_{n}\right) \notin K(a, R) \times K(b, R)\right\}$. We further partition $\mathcal{M}$ into the sets $\mathcal{M}_{k}$ as follows:

$$
\mathcal{M}_{k}=\left\{n: \max \left(\left\|a_{n}-a\right\|_{\infty},\left\|b_{n}-b\right\|_{\infty}\right) \in\left[2^{k} R, 2^{k+1} R\right)\right\}, \quad k \geq 0 .
$$

Since $D^{+}(\Lambda)<\infty$ by Lemma 2.1 (see also the remark after the lemma), we find that $\operatorname{card}\left(\mathcal{M}_{k}\right) \leq C_{1}\left(2^{k} R\right)^{2 d}$ for some large enough constant $C_{1}$. Thus

$$
\begin{aligned}
\sum_{m \in \mathcal{M}}\left|\left(\psi, f_{m}\right)\right|^{2} & =\sum_{k=0}^{\infty} \sum_{m \in \mathcal{M}_{k}}\left|\left(\psi, f_{m}\right)\right|^{2} \\
& \leq 2 \sum_{m \in \mathcal{M}}\left|\left(\eta, f_{m}\right)\right|^{2}+2 S \sum_{k=0}^{\infty} C_{1} 2^{2 k d} R^{2 d}\left(\left(2^{k}-1\right) R+R^{\delta}\right)^{-2 s} \\
& \leq 2 B\|\eta\|_{2}^{2}+2 S C_{1} R^{2 d-2 s \delta}+2 S C_{1} R^{2 d-2 s} \sum_{k=1}^{\infty} 2^{2 k d}\left(2^{k}-1\right)^{-2 s}
\end{aligned}
$$

where $B$ is the upper frame bound of $\left\{f_{n}\right\}_{n}$. Since $s>d$ by assumption, the last sum converges. Further, $s \delta>d$ and, by choosing $R$ large enough, the second 
and third terms can be made arbitrarily small. Given $\epsilon>0$ and $\delta \in(d / s, 1)$, we find that

$$
\sum_{m \in \mathcal{M}}\left|\left(\psi, f_{m}\right)\right|^{2} \leq C_{0}^{2} \epsilon^{2} \quad \text { for } R \geq R_{0}(\epsilon, \delta, \Lambda, S),
$$

with the constant $C_{0}$ depending only on the frame bound $B$ of $\left\{f_{n}\right\}_{n}$.

Step 2. Comparison with a basis of prolate spheroidal functions. For given $\epsilon>0$, $\delta \in(d / s, 1)$, and $R \geq R_{0}(\epsilon, \delta, \Lambda, S)$, we now consider those prolate spheroidal functions $\phi_{1}, \ldots, \phi_{N}$ with $N=N(R)$ that are $\epsilon d^{-1 / 2}$ concentrated on $(-R+$ $\left.R^{\delta}, R-R^{\delta}\right)$ and whose Fourier transforms are supported on $\left(-R+R^{\delta}, R-R^{\delta}\right)$. We refer the reader to [24] and [21] for definitions and properties of these functions. According to [21] the number of $\phi_{j}$ with these concentration properties satisfies $\lim _{R \rightarrow \infty} N(R) R^{-2}=1$.

In higher dimensions we take tensor products of phase space shifts of these prolate spheroidal functions. Let $\sigma=\left(n_{1}, \ldots, n_{d}\right) \in\{1,2, \ldots, N(R)\}^{d}$ and define

$$
\psi_{\sigma}(x)=\prod_{j=1}^{d} e^{-2 \pi i b_{j} x_{j}} \phi_{n_{j}}\left(x_{j}-a_{j}\right)
$$

then we obtain an orthonormal set of $N^{d}$ functions $\left\{\psi_{\sigma}\right\}_{\sigma}$ that are $\epsilon$-concentrated on $K\left(a, R-R^{\delta}\right)$ and whose Fourier transforms are supported on $K\left(b, R-R^{\delta}\right)$.

Now let $\left\{g_{n}\right\}_{n}$ be the dual frame of $\left\{f_{n}\right\}_{n}$. If $A>0$ is the lower frame bound of $\left\{f_{n}\right\}_{n}$, then we have

$$
\left\|\sum_{n} c_{n} g_{n}\right\|_{2}^{2} \leq A^{-1}\|c\|_{2}^{2} \quad \text { for every } c \in \ell^{2} .
$$

Step 3. Density estimate. We now follow the argument of Ramanathan and Steger in [23]. Let $S$ be the orthogonal projection of $L^{2}\left(\mathbb{R}^{d}\right)$ onto $\Psi=\operatorname{span}\left\{\psi_{\sigma}\right.$ : $\sigma \in\left\{1, \ldots, N(R)^{d}\right\}$ and let $T$ be the orthogonal projection onto $G=\operatorname{span}\left\{g_{n}\right.$ : $\left.n \in \mathcal{M}_{0}\right\}$. We consider $U: \Psi \rightarrow \Psi, U=S \circ T$. For each $\psi \in \Psi$ we obtain

$$
\begin{aligned}
\|\psi-U \psi\|_{2} & =\|S(\psi-T \psi)\|_{2} \leq\|\psi-T \psi\|_{2}=\inf _{g \in G}\|\psi-g\|_{2} \leq\left\|\psi-\sum_{n \in \mathcal{M}_{0}}\left(\psi, f_{n}\right) g_{n}\right\|_{2} \\
& =\left\|\sum_{m \in \mathcal{M}}\left(\psi, f_{m}\right) g_{m}\right\|_{2} \leq A^{-1 / 2}\left(\sum_{m \in \mathcal{M}}\left|\left(\psi, f_{m}\right)\right|^{2}\right)^{1 / 2} .
\end{aligned}
$$

Since each basis function $\psi_{\sigma}$ is in $\Psi$ and satisfies the concentration assumptions from Step 1, the estimate (2.1), implies that

$$
\left\|\psi_{\sigma}-U \psi_{\sigma}\right\|_{2} \leq \frac{C_{0}}{\sqrt{A}} \epsilon .
$$

Consequently,

$$
\operatorname{tr}(U) \geq \sum_{\sigma}\left(U \psi_{\sigma}, \psi_{\sigma}\right)=\sum_{\sigma}\left(\left\|\psi_{\sigma}\right\|_{2}^{2}-\left(\psi_{\sigma}-U \psi_{\sigma}, \psi_{\sigma}\right)\right) \geq\left(1-\frac{C_{0}}{\sqrt{A}} \epsilon\right) N(R)^{d}
$$


On the other hand, since $U$ is the composition of two projections, all eigenvalues of $U$ belong to $(0,1)$, and therefore $\operatorname{tr}(U) \leq \operatorname{rank}(U) \leq \operatorname{dim}(G)$. Thus

$$
\left(1-A^{-1 / 2} C_{0} \epsilon\right) N(R)^{d} \leq \operatorname{tr}(U) \leq \operatorname{card}(\Lambda \cap K(a, R) \times K(b, R)) .
$$

We now use the definition of the Beurling density with cubes in $\mathbb{R}^{2 d}$ instead of balls, and obtain

$$
\begin{aligned}
D^{-}(\Lambda) & =\lim _{R \rightarrow \infty} \inf _{(a, b) \in \mathbb{R}^{2 d}} \frac{\operatorname{card}(\Lambda \cap K(a, R) \times K(b, R))}{R^{2 d}} \\
& \geq\left(1-A^{-1 / 2} C_{0} \epsilon\right) \lim _{R \rightarrow \infty} \frac{N(R)^{d}}{R^{2 d}}=1-A^{-1 / 2} C_{0} \epsilon .
\end{aligned}
$$

As $\epsilon>0$ was arbitrary, we conclude that $D^{-}(\Lambda) \geq 1$.

Combining Lemmas 2.1 and 2.2, we obtain the density result for localized Riesz bases (recall, however, that our aim is to prove that there are no such bases).

Corollary. If $s>d$ and $\left\{f_{n}\right\}_{n=1}^{\infty}$ is a Riesz basis for $L^{2}\left(\mathbb{R}^{d}\right)$ that has phase space localization of magnitude $s$ at points $\left\{\left(a_{n}, b_{n}\right)\right\}_{n=1}^{\infty}$, then the density of $\Lambda=$ $\left\{\left(a_{n}, b_{n}\right)\right\}_{n}$ is $D(\Lambda)=D^{+}(\Lambda)=D^{-}(\Lambda)=1$.

\section{Uncertainty identity}

We first prove Theorem 1.1 under the additional condition that the dual basis is also well localized. The proof extends Battle's elegant proof of the Balian-Low theorem [3] and rediscovers Steger's argument mentioned by Bourgain in [7] (see the quote above).

The core of the argument is the following uncertainty identity (the canonical commutation relations)

$$
(x f, \nabla g)+(\nabla f, x g)=\sum_{j=1}^{d}\left(\left(x_{j} f, \frac{\partial g}{\partial x_{j}}\right)+\left(\frac{\partial f}{\partial x_{j}}, x_{j} g\right)\right)=-d(f, g),
$$

which holds provided that $f, g, \frac{\partial f}{\partial x_{j}}, \frac{\partial g}{\partial x_{j}}, x_{j} g, x_{j} f \in L^{2}\left(\mathbb{R}^{d}\right)$ for $j=1, \ldots, d$.

Lemma 3.1. Assume that $\left\{f_{n}\right\}_{n=1}^{\infty}$ is a Riesz basis for $L^{2}\left(\mathbb{R}^{d}\right)$ with the biorthogonal basis $\left\{g_{n}\right\}_{n=1}^{\infty}$. If the bases satisfy the localization estimates

(a) $\sup _{n} \int_{\mathbb{R}^{d}}\left|x-a_{n}\right|^{2 s}\left|f_{n}(x)\right|^{2} d x+\int_{\mathbb{R}^{d}}\left|\xi-b_{n}\right|^{2 s}\left|\widehat{f_{n}}(\xi)\right|^{2} d \xi=S^{2}<\infty$;

(b) $\sup _{n} \int_{\mathbb{R}^{d}}\left|x-a_{n}\right|^{2 s}\left|g_{n}(x)\right|^{2} d x+\int_{\mathbb{R}^{d}}\left|\xi-b_{n}\right|^{2 s}\left|\widehat{g_{n}}(\xi)\right|^{2} d \xi=T^{2}<\infty$; and

(c) $\Lambda=\left\{\left(a_{n}, b_{n}\right)\right\}_{n=1}^{\infty} \subset \mathbb{R}^{2 d}$ is relatively separated and

$$
0<D^{-}(\Lambda) \leq D^{+}(\Lambda)<\infty
$$

then $s \leq d$. 
Proof. We assume that $s>d$ and use the uncertainty identity to derive a contradiction from (a)-(c). In the following we will write $\overrightarrow{(x f, g)} \in \mathbb{C}^{d}$ for the vector with components $\left(x_{j} f, g\right), j=1, \ldots, d$. Likewise $\overrightarrow{(\nabla f, g)}=\left(\frac{\partial f}{\partial x_{j}}, g\right)_{j=1}^{d}$.

Step 1. An estimate for non-diagonal coefficients. Condition (a) implies that $x f_{n} \in L^{2}\left(\mathbb{R}^{d}\right)^{d}$, and then the sequence of vectors

$$
c_{m}^{n}=\overrightarrow{\left(x f_{n}, g_{m}\right)}=\left(x_{j} f_{n}, g_{m}\right)_{j=1}^{d} \in \mathbb{C}^{d}
$$

is well defined. By the biorthogonality condition, for $m \neq n$

$$
c_{m}^{n}=\overrightarrow{\left(\left(x-a_{n}\right) f_{n}, g_{m}\right)} .
$$

Since $\left\{g_{m}\right\}$ is a Riesz basis, assumption (a) implies that

$$
\sum_{m: m \neq n}\left|c_{m}^{n}\right|^{2} \leq B\left\|\left|x-a_{n}\right| f_{n}\right\|_{2}^{2} \leq B S^{2}
$$

Next, since $\overline{c_{m}^{n}}=\overrightarrow{\left(x g_{m}, f_{n}\right)}$, we also have

$$
\sum_{n: n \neq m}\left|c_{m}^{n}\right|^{2} \leq B\left\|\left|x-a_{m}\right| g_{m}\right\|_{2}^{2} \leq B T^{2} .
$$

Here $B$ is the upper basis constant for both Riesz bases $\left\{g_{m}\right\}_{m}$ and $\left\{f_{n}\right\}_{n}$.

The coefficients

$$
d_{m}^{n}=\widehat{\left(\widehat{\zeta f_{n}}, \widehat{g_{m}}\right)}=(2 \pi i)^{-1} \overrightarrow{\left(\nabla f_{n}, g_{m}\right)}
$$

enjoy similar properties.

Step 2. Commutation relations. We now apply the uncertainty identity to each pair $\left\{f_{n}, g_{n}\right\}$ and obtain

$$
\begin{aligned}
d & =-\sum_{m}\left(\overrightarrow{\left(x f_{n}, g_{m}\right)} \cdot \overrightarrow{\left(\nabla g_{n}, f_{m}\right)}+\overrightarrow{\left(\nabla f_{n}, g_{m}\right)} \cdot \overrightarrow{\left(x g_{n}, f_{m}\right)}\right) \\
& =-2 \pi i \sum_{m}\left(c_{m}^{n} \cdot \overline{d_{n}^{m}}-d_{m}^{n} \cdot \overline{c_{n}^{m}}\right)
\end{aligned}
$$

where $\lambda \cdot \mu=\sum_{j=1}^{d} \lambda_{j} \overline{\mu_{j}}$ is the standard scalar product in $\mathbb{C}^{d}$.

For each $R>0$ define $\mathcal{N}(R)=\left\{n:\left|a_{n}\right| \leq R,\left|b_{n}\right| \leq R\right\}$ and $N(R)=\operatorname{card} \mathcal{N}(R)$. Now we sum up the identities (3.1) for all $n \in \mathcal{N}(R)$,

$$
\frac{d}{2 \pi i} N(R)=\sum_{n \in \mathcal{N}(R)} \sum_{m}\left(-c_{m}^{n} \cdot \overline{d_{n}^{m}}+d_{m}^{n} \cdot \overline{c_{n}^{m}}\right)
$$

$=\sum_{n, m \in \mathcal{N}(R)} \sum_{j=1}^{d}\left(-\left(c_{m}^{n}\right)_{j}\left(d_{n}^{m}\right)_{j}+\left(d_{m}^{n}\right)_{j}\left(c_{n}^{m}\right)_{j}\right)+\sum_{n \in \mathcal{N}(R)} \sum_{m \notin \mathcal{N}(R)}\left(-c_{m}^{n} \cdot \overline{d_{n}^{m}}+d_{m}^{n} \cdot \overline{c_{n}^{m}}\right)$.

Clearly, the first sum equals zero. We will derive a contradiction by showing that the second sum grows more slowly than $N(R)$. We divide the necessary estimates into several steps. 
Step 3. Points $\left(a_{n}, b_{n}\right)$ near the boundary. To estimate the second sum, we partition $\mathcal{N}(R)$ into two sets,

$$
\mathcal{N}(R)=\mathcal{N}(R-r) \cup(\mathcal{N}(R) \backslash \mathcal{N}(R-r)),
$$

where $r=R^{\delta}$ for some $\delta \in(d / s, 1)$.

First, for $n \in \mathcal{R}(R-r, R)=\mathcal{N}(R) \backslash \mathcal{N}(R-r)$ we get

$$
\begin{aligned}
& \text { (3.3) } \sum_{n \in \mathcal{R}(R-r, R)} \sum_{m \notin \mathcal{N}(R)}\left|c_{m}^{n}\right|\left|d_{n}^{m}\right| \\
& \leq \sum_{n \in \mathcal{R}(R-r, R)}\left(\sum_{m: m \neq n}\left|c_{m}^{n}\right|^{2}\right)^{1 / 2}\left(\sum_{m: m \neq n}\left|d_{n}^{m}\right|^{2}\right)^{1 / 2} \leq(N(R)-N(R-r)) B S T .
\end{aligned}
$$

The sum of $\left|d_{m}^{n} \| c_{n}^{m}\right|$ admits the same estimate.

Step 4. Further partition of $\mathcal{N}(R)$ for interior $\left(a_{n}, b_{n}\right)$. Next we partition the complement of $\mathcal{N}(R)$ into the rings $\mathcal{N}_{k}=\mathcal{N}\left(R_{k+1}\right) \backslash \mathcal{N}\left(R_{k}\right)$ where $R_{k}=2^{k} R$, $k \geq 0$. Then for each $n \in \mathcal{N}(R-r)$ we get

$$
\begin{aligned}
\sum_{m \notin \mathcal{N}(R)}\left|c_{m}^{n}\right|\left|d_{n}^{m}\right|= & \sum_{k=0}^{\infty} \sum_{m \in \mathcal{N}_{k}}\left|c_{m}^{n}\right|\left|d_{n}^{m}\right| \\
\leq & \sum_{k=0}^{\infty} \sum_{m \in \mathcal{N}_{k}:\left|a_{m}\right|>R_{k}}\left|c_{m}^{n}\right|\left|d_{n}^{m}\right|+\sum_{k=0}^{\infty} \sum_{m \in \mathcal{N}_{k}:\left|b_{m}\right|>R_{k}}\left|c_{m}^{n}\right|\left|d_{n}^{m}\right| \\
\leq & \left(\sum_{k=0}^{\infty} \sum_{m \in \mathcal{N}_{k}:\left|a_{m}\right|>R_{k}}\left|c_{m}^{n}\right|^{2}\right)^{1 / 2}\left(\sum_{m: m \neq n}\left|d_{n}^{m}\right|^{2}\right)^{1 / 2} \\
& +\left(\sum_{m: m \neq n}\left|c_{m}^{n}\right|^{2}\right)^{1 / 2}\left(\sum_{k=0}^{\infty} \sum_{m \in \mathcal{N}_{k}:\left|b_{m}\right|>R_{k}}\left|d_{n}^{m}\right|^{2}\right)^{1 / 2}
\end{aligned}
$$

Step 5. Main estimate. Now we write down an estimate for $c_{m}^{n}$ when $\left|a_{n}\right|<R-r$ and $\left|a_{m}\right|>R_{k}$. Set

$$
h_{n}^{(j)}(x)=\left(x-a_{n}\right)_{j} f_{n}(x)\left(1-\chi_{B(R-r / 2)}(x)\right), \quad j=1,2, \ldots, d .
$$

Then

$$
\left\|h_{n}^{(j)}\right\|_{2}^{2} \leq \int_{|x|>R-r / 2}\left|\left(x-a_{n}\right)_{j}\right|^{2}\left|f_{n}(x)\right|^{2} d x .
$$

Further, for $|x| \geq r / 2$ and $\left|a_{n}\right| \leq R-r$, we have $\left|x-a_{n}\right| \geq r / 2$, and therefore

$$
\sum_{j=1}^{d}\left\|h_{n}^{(j)}\right\|_{2}^{2} \leq(r / 2)^{2-2 s} \int_{\mathbb{R}^{d}}\left|x-a_{n}\right|^{2 s}\left|f_{n}(x)\right|^{2} d x \leq(r / 2)^{2-2 s} S^{2} .
$$


Then we have

$$
\begin{aligned}
\left|c_{m}^{n}\right|^{2} & =\sum_{j=1}^{d}\left|\left(\left(x-a_{n}\right)_{j} f_{n}, g_{m}\right)\right|^{2} \\
& \leq \sum_{j=1}^{d}\left(2\left|\left(\left(x-a_{n}\right)_{j} f_{n} \chi_{B(R-r / 2)}, g_{m}\right)\right|^{2}+2\left|\left(h_{n}^{(j)}, g_{m}\right)\right|^{2}\right) \\
& \leq 2 S^{2}\left\|g_{m} \chi_{B(R-r / 2)}\right\|_{2}^{2}+2 \sum_{j=1}^{d}\left|\left(h_{n}^{(j)}, g_{m}\right)\right|^{2} \\
& \leq 2 S^{2} T^{2}\left(R_{k}-R+r / 2\right)^{-2 s}+2 \sum_{j=1}^{d}\left|\left(h_{n}^{(j)}, g_{m}\right)\right|^{2} .
\end{aligned}
$$

And since $\left\{g_{m}\right\}_{m}$ is a Riesz basis,

$$
\sum_{m}\left|\left(h_{n}^{(j)}, g_{m}\right)\right|^{2} \leq B\left\|h_{n}^{(j)}\right\|_{2}^{2}
$$

Summing up the estimates (3.5) over all $k$ and all $m \in \mathcal{N}_{k}$ such that $\left|a_{m}\right|>R_{k}$ and taking into account (3.4) and (3.6), we obtain

$$
\begin{aligned}
& \sum_{k=0}^{\infty} \sum_{m \in \mathcal{N}_{k}:\left|a_{m}\right|>R_{k}}\left|c_{m}^{n}\right|^{2} \\
& \quad \leq 2 S^{2} T^{2} \sum_{k=0}^{\infty} N\left(R_{k+1}\right)\left(R_{k}-R+r / 2\right)^{-2 s}+B(r / 2)^{2-2 s} S^{2}
\end{aligned}
$$

We can derive similar estimates of $\sum_{k} \sum_{m}\left|d_{n}^{m}\right|^{2}$ where the summation is over all $k$ and $m \in \mathcal{N}_{k}$ such that $\left|b_{m}\right|>R_{k}$ by using the localization inequality for $\widehat{g_{n}}$. Likewise, we obtain the estimates for $\sum_{k} \sum_{m}\left|c_{n}^{m}\right|^{2}$ and $\sum_{k} \sum_{m}\left|d_{m}^{n}\right|^{2}$, where the sums are over all $k$ and $m \in \mathcal{N}_{k}$ such that $\left|b_{m}\right|>R_{k}$ and over all $k$ and $m \in \mathcal{N}_{k}$ such that $\left|a_{m}\right|>R_{k}$, by using the localization conditions on $g_{n}$ and $\widehat{f_{n}}$.

Step 6. Comparison of the densities. Finally we combine the inequality obtained in Step 4 with the last inequality of Step 5 and similar inequalities with other combinations of indices. Then we obtain for every $n \in \mathcal{N}(R-r)$

$$
\sum_{m \notin \mathcal{N}(R)}\left(\left|c_{m}^{n}\right|\left|d_{m}^{n}\right|+\left|c_{n}^{m}\right|\left|d_{n}^{m}\right|\right) \leq\left(C_{1} r^{2-2 s}+C_{2} \sum_{k=0}^{\infty} N\left(R_{k+1}\right)\left(R_{k}-R+r / 2\right)^{-2 s}\right)^{1 / 2}
$$

where $C_{1}$ and $C_{2}$ depend on $S, T, B, s$, and $\sup _{n}\left\|f_{n}\right\|_{2}$ and $\sup _{n}\left\|g_{n}\right\|_{2}$. Assumption (c) (the estimate of the upper density $D^{+}(\Lambda)<\infty$ ) implies that

$$
N\left(R_{k+1}\right) \leq D_{1} 2^{2 d(k+1)} R^{2 d} \quad \text { and } \quad N(R-r) \leq D_{1}(R-r)^{2 d}
$$


for some $D_{1}>0$ and all large enough $R$. Then for large enough $R$ we obtain

$$
\begin{aligned}
& \sum_{n \in \mathcal{N}(R-r)} \sum_{m \notin \mathcal{N}(R)}\left(\left|c_{m}^{n}\right|\left|d_{n}^{m}\right|+\left|c_{n}^{m}\right|\left|d_{m}^{n}\right|\right) \\
& \leq C N(R-r)\left(r^{2-2 s}+R^{2 d}(r / 2)^{-2 s}+R^{2 d} \sum_{k=1}^{\infty} 2^{2 d(k+1)}\left(2^{k} R-R+r / 2\right)^{-2 s}\right)^{1 / 2} \\
& \leq C(R-r)^{2 d} R^{d} r^{-s}
\end{aligned}
$$

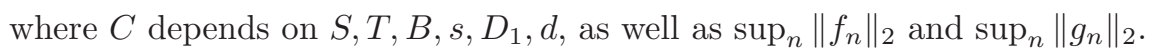

To finish the proof we recall that $r=R^{\delta}$ and $\delta \in(d / s, 1)$. Observe that for $r$ large enough the estimate of the upper density implies $N(R)-N(R-r) \leq$ $D_{1} R^{2 d-1} r$ (we just cover the set $Q(0, R) \backslash Q(0, R-r)$ by cubes with side length $r$ ). Now, combining (3.2), (3.3), and the last inequality, we obtain

$$
N(R) \leq C_{3}\left(N(R)-N(R-r)+R^{3 d-\delta s}\right) \leq C_{4}\left(R^{2 d-1+\delta}+R^{3 d-\delta s}\right) .
$$

If we now let $R$ go to infinity, we see that

$$
D^{-}(\Lambda) \leq \lim _{R \rightarrow \infty} \frac{N(R)}{R^{2 d}} \leq C_{4} \lim _{R \rightarrow \infty}\left(R^{\delta-1}+R^{d-\delta s}\right)=0 .
$$

This conclusion contradicts the assumption $(c)$ that the lower density estimate $D^{-}(\Lambda)$ is strictly positive.

Lemma 3.1 concludes the proof of Theorem 1.1 for the case of an orthonormal basis. For a Riesz basis we are not able to prove that the phase space localization of magnitude $s$ (condition (a) of the lemma) with $s>d$ implies the required localization for its biorthogonal basis (stated in (b)). For the general case a more complicated argument is presented in the next sections.

\section{Some preliminaries on modulation spaces}

The proof of Theorem 1.1 for Riesz bases requires some tools from time-frequency analysis. We give a minimalistic account of the required facts on the short time Fourier transform and modulation spaces. The reader can find the details and a much more general theory of modulation spaces in [14].

Short time Fourier Transform. For $(a, b) \in \mathbb{R}^{2 d}$ we write

$$
\pi(a, b) f(t)=e^{2 \pi i b \cdot t} f(t-a)
$$

for the phase space shift of a function $f$ on $\mathbb{R}^{d}$. Let $g(x)=2^{-d / 4} e^{-\pi|x|^{2}}$ be the normalized Gaussian function on $\mathbb{R}^{d}$. We consider the short time Fourier transform of a function $\phi \in L^{2}\left(\mathbb{R}^{d}\right)$ with respect to $g$ (see Chapter 3 of [14]),

$$
V_{g} \phi(x, \xi)=(\phi, \pi(x, \xi) g)=\int_{\mathbb{R}^{d}} \phi(t) \overline{g(t-x)} e^{-2 \pi i t \cdot \xi} d t, \quad x, \xi \in \mathbb{R}^{d} .
$$


The inversion formula for the short time Fourier transform yields

$$
\phi(t)=\iint_{\mathbb{R}^{2 d}} V_{g} \phi(x, \xi) e^{2 \pi i \xi \cdot t} g(t-x) d x d \xi,
$$

for every $\phi \in L^{2}\left(\mathbb{R}^{d}\right)$, with a weak interpretation of the vector-valued integral.

Modulation spaces. For each $s \geq 0$ let

$$
L_{s}^{2}\left(\mathbb{R}^{m}\right)=\left\{f \in L^{2}\left(\mathbb{R}^{m}\right):\|f\|_{L_{s}^{2}}^{2}=\int_{\mathbb{R}^{m}}|f(x)|^{2}(1+|x|)^{2 s} d x<\infty\right\} .
$$

The modulation space $M_{s}^{2}\left(\mathbb{R}^{d}\right)$ is defined by

$$
M_{s}^{2}\left(\mathbb{R}^{d}\right)=\left\{\phi \in L^{2}\left(\mathbb{R}^{d}\right):\|\phi\|_{M_{s}^{2}}=\left\|V_{g} \phi\right\|_{L_{s}^{2}\left(\mathbb{R}^{2 d}\right)}<\infty\right\} .
$$

The following norm equivalence identifies the modulation space $M_{s}^{2}$ with the Fourier-Lebesgue space $L_{s}^{2} \cap \mathcal{F} L_{s}^{2}$ (see Propositions 11.3.1 and 12.1.6 of [14]):

$$
c_{1}\|\phi\|_{M_{s}^{2}} \leq\|\phi\|_{L_{s}^{2}}+\|\widehat{\phi}\|_{L_{s}^{2}} \leq c_{2}\|\phi\|_{M_{s}^{2}} .
$$

The adjoint operator $V_{g}^{*}$ of the short time Fourier transform is defined on $L^{2}\left(\mathbb{R}^{2 d}\right)$ by

$$
V_{g}^{*} F(t)=\iint_{\mathbb{R}^{2 d}} F(x, \xi) e^{2 \pi i \xi \cdot t} g(t-x) d x d \xi=\iint_{\mathbb{R}^{2 d}} F(x, \xi) \pi(x, \xi) g(t) d x d \xi .
$$

If $F \in L_{s}^{2}\left(\mathbb{R}^{2 d}\right)$, then by Proposition 11.3.2 in [14], $V_{g}^{*} F \in M_{s}^{2}\left(\mathbb{R}^{d}\right)$ and

$$
\left\|V_{g}^{*} F\right\|_{M_{s}^{2}} \leq C\|F\|_{L_{s}^{2}} .
$$

We note that the phase space localization of magnitude $s$ can be rephrased as

$$
\sup _{n \in \mathbb{N}} \inf _{(a, b) \in \mathbb{R}^{2 d}}\left\|\pi(a, b) f_{n}\right\|_{M_{s}^{2}}<\infty .
$$

Amalgam spaces. We define the amalgam space $W\left(L_{s}^{2}\right) \subset L_{s}^{2}\left(\mathbb{R}^{2 d}\right) \cap L^{\infty}\left(\mathbb{R}^{2 d}\right)$ as the space of all continuous functions on $\mathbb{R}^{2 d}$ for which the norm

$$
\|F\|_{W\left(L_{s}^{2}\right)}^{2}=\sum_{k, n \in \mathbb{Z}^{d}} \sup _{x, \xi \in[0,1]^{d}}|F(x+k, \xi+n)|^{2}(1+|k|+|n|)^{2 s}
$$

is finite. For $s=0,\|F\|_{W\left(L^{2}\right)} \geq\|F\|_{2}$ obviously. The continuity of $F$ implies the existence of points $x_{k n}, \xi_{k n} \in[0,1]^{d}$, such that

$$
\|F\|_{W\left(L_{s}^{2}\right)}^{2}=\sum_{k, n \in \mathbb{Z}^{d}}\left|F\left(k+x_{k n}, n+\xi_{k n}\right)\right|^{2}(1+|k|+|n|)^{2 s} .
$$

The definition of $W\left(L_{s}^{2}\right)$ implies the following sampling inequality: If $\Lambda=\left\{\lambda_{n}\right\} \subseteq$ $\mathbb{R}^{2 d}$ is relatively separated, $z \in \mathbb{R}^{2 d}$, and $F \in W\left(L_{s}^{2}\right)$, then

$$
\left(\sum_{n}\left|F\left(z+\lambda_{n}\right)\right|^{2}\left(1+\left|z+\lambda_{n}\right|\right)^{2 s}\right)^{1 / 2} \leq \sup _{k \in \mathbb{Z}^{2 d}} \operatorname{card}\left(\Lambda \cap\left(k+[0,1]^{2 d}\right)\right)\|F\|_{W_{L_{s}^{2}}} .
$$

The following important inequality links modulation spaces with amalgam spaces: For every $\phi \in L_{s}^{2}\left(\mathbb{R}^{d}\right)$ with $\widehat{\phi} \in L_{s}^{2}\left(\mathbb{R}^{d}\right)$ we have, e.g., by Theorem 12.2 .1 of [14],

$$
\left\|V_{g} \phi\right\|_{W\left(L_{s}^{2}\right)} \leq C\left\|V_{g} \phi\right\|_{L_{s}^{2}}=C\|\phi\|_{M_{s}^{2}} .
$$




\section{Basis modification}

To finish the proof of Theorem 1.1, we will modify a given Riesz basis $\left\{f_{n}\right\}_{n}$ for $L^{2}\left(\mathbb{R}^{d}\right)$ that has phase space localization of magnitude $s>d$ into a Riesz basis with much better localization properties. The argument in this section may be of independent interest and can also be used to prove positive results about frames and bases.

Proposition 5.1. Assume that $\left\{f_{n}\right\}_{n=1}^{\infty}$ is a Riesz basis for $L^{2}\left(\mathbb{R}^{d}\right)$ that satisfies

$$
\sup _{n \in \mathbb{N}}\left(\int_{\mathbb{R}^{d}}\left|x-a_{n}\right|^{2 s}\left|f_{n}(x)\right|^{2}+\int_{\mathbb{R}^{d}}\left|\xi-b_{n}\right|^{2 s}\left|\widehat{f_{n}}(\xi)\right|^{2}\right)<\infty
$$

for some $s>d$. Then there exists a Riesz basis $\left\{h_{n}\right\}_{n=1}^{\infty}$ that satisfies

$$
\sup _{n \in \mathbb{N}}\left(\int_{\mathbb{R}^{d}}\left|x-a_{n}\right|^{2 t}\left|h_{n}(x)\right|^{2}+\int_{\mathbb{R}^{d}}\left|\xi-b_{n}\right|^{2 t}\left|\widehat{h_{n}}(\xi)\right|^{2}\right)<\infty
$$

for every $t>0$.

Proof. The new basis is obtained by a modification of $\left\{f_{n}\right\}_{n}$. We use the inversion formula for the short time Fourier transform and truncate it. In the language of time-frequency analysis we apply a localization operator to $f_{n}$. Precisely, let $R>0$ and $Q(R)=Q(0, R)=B(0, R) \times B(0, R) \subset \mathbb{R}^{2 d}$. Then the localization operator $A_{R}$ is defined by

$$
A_{R} f(t)=\iint_{Q(R)} V_{g} f(x, \xi) e^{2 \pi i \xi \cdot t} g(t-x) d x d \xi, \quad f \in L^{2}\left(\mathbb{R}^{d}\right) .
$$

Intuitively, $A_{R} f$ is the part of $f$ that is concentrated on the set $Q(R)$ in the phase space. For more on localization operators see for instance [26] and [8].

We recast the assumption as follows: $\left\{f_{n}\right\}_{n}$ is a Riesz basis for $L^{2}\left(\mathbb{R}^{d}\right), f_{n}(x)=$ $e^{2 \pi i b_{n} x} \phi_{n}\left(x-a_{n}\right), s>d$ and

$$
\sup _{n \in \mathbb{N}}\left\|\phi_{n}\right\|_{M_{s}^{2}}^{2} \leq S^{2}<\infty
$$

We now define

$$
\psi_{n}=A_{R} \phi_{n}=\iint_{Q(R)} V_{g} \phi_{n}(x, \xi) \pi(x, \xi) g d x d \xi
$$

and the modified basis $h_{n}(x)=h_{n}^{R}(x)=e^{2 \pi i b_{n} x} \psi_{n}\left(x-a_{n}\right)$.

Claim. For $R$ large enough $\left\{h_{n}\right\}_{n=1}^{\infty}$ is a Riesz basis for $L^{2}\left(\mathbb{R}^{d}\right)$.

To prove the claim, it suffices to show that for every $\epsilon>0$ there exists $R$ such that for $h_{n}=h_{n}^{R}$ and every sequence $c=\left\{c_{n}\right\}_{n} \in \ell^{2}$ the inequality

$$
\left\|\sum_{n} c_{n}\left(f_{n}-h_{n}\right)\right\|_{2} \leq \epsilon\|c\|_{2}
$$


holds. If $\left\{f_{n}\right\}_{n}$ is a Riesz basis with the lower basis constant $A>0$, then

$$
\left\|\sum_{n} c_{n} h_{n}\right\|_{2} \geq\left\|\sum_{n} c_{n} f_{n}\right\|_{2}-\left\|\sum_{n} c_{n}\left(f_{n}-h_{n}\right)\right\|_{2} \geq(A-\epsilon)\|c\|_{2},
$$

and so $\left\{h_{n}\right\}_{n}$ is a Riesz basis.

Using once again the crucial assumption $s>d$, we now choose a number $\sigma$ such that $d<\sigma<s$. Using the inversion formula for the short time Fourier transform and (5.3), we write

$$
\phi_{n}-\psi_{n}=\iint_{\mathbb{R}^{2 d}}\left(1-\chi_{Q(R)}(x, \xi)\right) V_{g} \phi_{n}(x, \xi) \pi(x, \xi) g d x d \xi,
$$

and estimate the $M_{\sigma}^{2}$-norm of $\phi_{n}-\psi_{n}$ with (4.1) as

$$
\begin{aligned}
\| \phi_{n}- & \psi_{n} \|_{M_{\sigma}^{2}}^{2} \leq \iint_{\mathbb{R}^{2 d}}\left(1-\chi_{Q(R)}(x, \xi)\right)\left|V_{g} \phi_{n}(x, \xi)\right|^{2}(1+|x|+|\xi|)^{2 \sigma} d x d \xi \\
& \leq(1+R)^{2(\sigma-s)} \iint_{\mathbb{R}^{2 d}}\left(1-\chi_{Q(R)}(x, \xi)\right)\left|V_{g} \phi_{n}(x, \xi)\right|^{2}(1+|x|+|\xi|)^{2 s} d x d \xi \\
& \leq C(1+R)^{2(\sigma-s)}\left\|\phi_{n}\right\|_{M_{s}^{2}}^{2} \leq C(1+R)^{2(\sigma-s)} S^{2} .
\end{aligned}
$$

Choosing now $R$ large enough, we have

$$
\left\|\phi_{n}-\psi_{n}\right\|_{M_{\sigma}^{2}}<\epsilon \text { for all } n \text {. }
$$

Then we have, with (4.2) and a suitable choice of points $\left(x_{k, m}, \xi_{k, m}\right) \in[0,1]^{2 d}$, that

$$
\begin{aligned}
\| & \sum_{n} c_{n}\left(f_{n}-h_{n}\right)\left\|_{2}^{2}=\right\| \sum_{n} c_{n} V_{g}\left(f_{n}-h_{n}\right)\left\|_{2}^{2} \leq\right\| \sum_{n} c_{n} V_{g}\left(f_{n}-h_{n}\right) \|_{W\left(L^{2}\right)}^{2} \\
= & \sum_{(k, m) \in \mathbb{Z}^{2 d}}\left|\sum_{n} c_{n} V_{g}\left(f_{n}-h_{n}\right)\left(x_{k, m}+k, \xi_{k, m}+m\right)\right|^{2} \\
\leq & \sum_{(k, m) \in \mathbb{Z}^{2 d}}\left(\sum_{n}\left|c_{n}\right|\left|V_{g}\left(f_{n}-g_{n}\right)\left(x_{k, m}+k, \xi_{k, m}+m\right)\right|\right)^{2} \\
\leq & \sum_{(k, m) \in \mathbb{Z}^{2 d}}\left(\sum_{n}\left(1+\left|k-a_{n}\right|+\left|m-b_{n}\right|\right)^{-2 \sigma}\right) \\
& \cdot\left(\sum_{n}\left|c_{n}\right|^{2}\left|V_{g}\left(f_{n}-g_{n}\right)\left(x_{k, m}+k, \xi_{k, m}+m\right)\right|^{2}\left(1+\left|k-a_{n}\right|+\left|m-b_{n}\right|\right)^{2 \sigma}\right) .
\end{aligned}
$$

Since $\sigma>d$ and $\Lambda$ is relatively separated, the sum $\sum_{n}\left(1+\left|k-a_{n}\right|+\left|m-b_{n}\right|\right)^{-2 \sigma}$ is uniformly bounded, independent of $k$ and $m$. Thus we obtain

$$
\begin{aligned}
& \left\|\sum_{n} c_{n}\left(f_{n}-h_{n}\right)\right\|_{2}^{2} \\
& \quad \leq C \sum_{n}\left|c_{n}\right|^{2} \sum_{k, m}\left|V_{g}\left(f_{n}-g_{n}\right)\left(x_{k, m}+k, \xi_{k, m}+m\right)\right|^{2}\left(1+\left|k-a_{n}\right|+\left|m-b_{n}\right|\right)^{2 \sigma} .
\end{aligned}
$$


By (4.3) and (4.4) we estimate further that

$$
\begin{aligned}
& \sum_{k, m}\left|V_{g}\left(f_{n}-g_{n}\right)\left(x_{k, m}+k, \xi_{k, m}+m\right)\right|^{2}\left(1+\left|k-a_{n}\right|+\left|m-b_{n}\right|\right)^{2 \sigma} \\
& \quad=\sum_{k, m}\left|V_{g}\left(\phi_{n}-\psi_{n}\right)\left(x_{k, m}+k-a_{n}, \xi_{k, m}+m-b_{n}\right)\right|^{2}\left(1+\left|k-a_{n}\right|+\left|m-b_{n}\right|\right)^{2 \sigma} \\
& \quad \leq C_{2}\left\|V_{g}\left(\phi_{n}-\psi_{n}\right)\right\|_{W\left(L_{\sigma}^{2}\right)}^{2} \leq C_{3}\left\|\phi_{n}-\psi_{n}\right\|_{M_{\sigma}^{2}}^{2}<C_{3} \epsilon^{2} .
\end{aligned}
$$

Collecting all these estimates, we arrive at

$$
\left\|\sum_{n} c_{n}\left(f_{n}-h_{n}\right)\right\|_{2}^{2} \leq C_{3} \epsilon^{2} \sum_{n}\left|c_{n}\right|^{2}=C_{3}\|c\|_{2}^{2} \epsilon^{2} .
$$

Consequently, $\left\{h_{n}\right\}_{n}$ is a Riesz basis for $L^{2}\left(\mathbb{R}^{d}\right)$.

Finally, applying (4.1) once again, we obtain, for arbitrary $t>0$,

$$
\left\|\psi_{n}\right\|_{L_{t}^{2}}+\left\|\hat{\psi}_{n}\right\|_{L_{t}^{2}} \leq C\left\|\psi_{n}\right\|_{M_{t}^{2}} \leq C^{\prime}\left\|V_{g} \phi_{n} \chi_{Q(R)}\right\|_{L_{t}^{2}} \leq C_{t} R^{t},
$$

which is $(5.2)$.

Remark. The construction of $\psi_{n}$ implies that $\left|\psi_{n}(t)\right| \leq C e^{-\alpha|t|^{2}}$ and $\left|\widehat{\psi_{n}}(\xi)\right| \leq$ $C e^{-\beta|\xi|^{2}}$ for some $\alpha, \beta, C>0$. Thus the perturbed basis belongs to the GelfandShilov space $S^{1 / 2,1 / 2}$, the smallest space of test functions that is invariant under the Fourier transform.

To complete the proof of Theorem 1.1 we will show that the biorthogonal basis $\left\{\widetilde{h_{n}}\right\}_{n}$ satisfies (5.1) for some $s$ large enough and then apply Lemma 3.1.

The modified basis $\left\{h_{n}\right\}_{n}$ possesses enough phase space localization so that the theory of localized frames [12], [15] is applicable. We say that a frame $\left\{h_{\lambda}: \lambda \in \Lambda\right\}$ is $s$-localized over the index set $\Lambda \subseteq \mathbb{R}^{d}$, if its Gramian satisfies

$$
\left|\left(h_{\mu}, h_{\lambda}\right)\right| \leq C(1+|\lambda-\mu|)^{-s} \quad \text { for all } \lambda, \mu \in \Lambda \text {. }
$$

The main result about localized frames asserts that the dual frame possesses the same type of localization. Specifically we need the following result taken from Theorem 1.1 and Corollary 3.7 of [12]:

Proposition 5.2. Let $\Lambda \subseteq \mathbb{R}^{2 d}$ be a relatively separated set and let $\left\{h_{\lambda}: \lambda \in \Lambda\right\}$ be a frame for $L^{2}\left(\mathbb{R}^{d}\right)$. Assume that $\left\{h_{\lambda}\right\}$ is $s$-localized for $s>2 d$. Then the (canonical) dual frame is also s-localized, i.e.,

$$
\left|\left(\widetilde{h_{\mu}}, \widetilde{h_{\lambda}}\right)\right| \leq C^{\prime}(1+|\lambda-\mu|)^{-s} \quad \text { for all } \lambda, \mu \in \Lambda \text {. }
$$

For a Riesz basis this result can be proved directly. The Gramian matrix $G$ of the basis with entries $\left(h_{\mu}, h_{\lambda}\right)$ is invertible with inverse $\left(G^{-1}\right)_{\lambda \mu}=\left(\widetilde{h_{\mu}}, \widetilde{h_{\lambda}}\right)$. By a theorem of Jaffard [18] the polynomial off-diagonal decay is preserved under inversion, whence follows the statement of the proposition (for a Riesz basis).

To apply Proposition 5.2, we need to compare the phase space localization of magnitude $s$ in Bourgain's uncertainty principle with the localization defined by the off-diagonal decay of the Gramian. 
Lemma 5.3. (i) If $\left\{h_{n}\right\}_{n}$ is a Riesz basis with phase space localization of magnitude $s>0$, then $\left\{h_{n}\right\}_{n}$ is s-localized over the index set $\Lambda$ in the sense of (5.4).

(ii) If $\left\{h_{n}\right\}_{n}$ is a Riesz basis with phase space localization of magnitude $s>3 d$, then the biorthogonal basis $\left\{\widetilde{h_{n}}\right\}_{n}$ has phase space localization of magnitude $t$ for any $t \in(d, s-2 d)$.

Proof. (i) We choose the set $\Lambda=\left\{\left(a_{n}, b_{n}\right)\right\}_{n} \subset \mathbb{R}^{2 d}$ as the appropriate index set. By Lemma 2.1, $\Lambda$ is relatively separated. Then the inequality

$$
\left(1+\left|x-a_{m}\right|\right)^{s}\left(1+\left|x-a_{n}\right|\right)^{s} \geq\left(1+\left|a_{m}-a_{n}\right|\right)^{s}
$$

implies

$$
\begin{aligned}
\left|\left(h_{n}, h_{m}\right)\right| & \leq\left(1+\left|a_{m}-a_{n}\right|\right)^{-s} \int_{\mathbb{R}^{d}} h_{n}(x)\left(1+\left|x-a_{n}\right|\right)^{s} \overline{h_{m}(x)}\left(1+\left|x-a_{m}\right|\right)^{s} d x \\
& \leq\left(1+\left|a_{m}-a_{n}\right|\right)^{-s} \sup _{n \in \mathbb{N}} \int_{\mathbb{R}^{d}}\left|h_{n}(x)\right|^{2}\left(1+\left|x-a_{n}\right|\right)^{2 s} d x \\
& =\left(1+\left|a_{m}-a_{n}\right|\right)^{-s} S^{2} .
\end{aligned}
$$

The argument for the Fourier transforms yields that

$$
\left|\left(h_{n}, h_{m}\right)\right|=\left|\left(\widehat{h_{n}}, \widehat{h_{m}}\right)\right| \leq\left(1+\left|b_{m}-b_{n}\right|\right)^{-s} S^{2} .
$$

By combining both estimates we arrive at

$$
\left|\left(h_{n}, h_{m}\right)\right| \leq C(1+|\lambda-\mu|)^{-s} \quad \text { for all } \lambda, \mu \in \Lambda,
$$

in other words, $\left\{h_{n}\right\}$ is $s$-localized over $\Lambda$.

(ii) By (i), $\left\{h_{n}\right\}$ is $s$-localized on $\Lambda \subseteq \mathbb{R}^{2 d}$ and $s>3 d$. Therefore Proposition 5.2 applies and the dual frame is also $s$-localized as in (5.5). Now we have

$$
\widetilde{h_{n}}=\sum_{m}\left(\widetilde{h_{n}}, \widetilde{h_{m}}\right) h_{m}
$$

Next let $d<t<s-2 d$ (which is possible by $s>3 d$ ). A straightforward calculation and (5.2) give

$$
\begin{aligned}
\int_{\mathbb{R}^{d}}\left|x-a_{n}\right|^{2 t}\left|h_{m}(x)\right|^{2} d x & \leq \int_{\mathbb{R}^{d}} C_{t}\left(\left|x-a_{m}\right|^{2 t}+\left|a_{n}-a_{m}\right|^{2 t}\right)\left|h_{m}(x)\right|^{2} d x \\
& \leq C\left(S+\left|a_{n}-a_{m}\right|^{2 t}\right) \leq C^{\prime}\left(1+\left|\lambda_{n}-\lambda_{m}\right|\right)^{2 t} .
\end{aligned}
$$

With the triangle inequality for the $L_{t}^{2}$-norm we obtain

$$
\begin{aligned}
\left(\int_{\mathbb{R}^{d}}\left|x-a_{n}\right|^{2 t}\right. & \left.\left|\tilde{h}_{n}(x)\right|^{2} d x\right)^{1 / 2}=\left(\int_{\mathbb{R}^{d}}\left|\sum_{m}\left(\tilde{h}_{n}, \tilde{h}_{m}\right) h_{m}(x)\right|^{2}\left|x-a_{n}\right|^{2 t} d x\right)^{1 / 2} \\
& \leq C \sum_{m}\left|\left(\tilde{h}_{n}, \tilde{h}_{m}\right)\right|\left(1+\left|\lambda_{n}-\lambda_{m}\right|\right)^{t} \leq C \sum_{m}\left(1+\left|\lambda_{n}-\lambda_{m}\right|\right)^{t-s} .
\end{aligned}
$$

Since $\Lambda$ is relatively separated and $t-s<-2 d$, the last sum is uniformly bounded.

Similar estimates hold for the Fourier transforms. Consequently, the dual basis is localized in the sense of (5.1) for $t \in(d, s-2 d)$. 
We now can finish the proof of Theorem 1.1 for Riesz bases.

We start with a Riesz basis $\left\{f_{n}\right\}_{n}$ that satisfies (5.1) for some $s>d$. Then we modify this basis by means of Proposition 5.1 to a Riesz basis $\left\{h_{n}\right\}_{n}$ that satisfies the localization estimates (5.1) for all $t>0$. Finally, Lemma 5.3 guarantees that the dual basis $\left\{\widetilde{h_{n}}\right\}_{n}$ also satisfies the localization estimates (5.1) for all $t>0$. Thus all the assumptions of Lemma 3.1 are satisfied whence we conclude that the localization of $\left\{h_{n}\right\}_{n}$ is $t \leq d$. This is a contradiction to the construction of $\left\{h_{n}\right\}_{n}$. This means that the original basis $\left\{f_{n}\right\}_{n}$ cannot satisfy the strong localization estimate $s>d$, and the proof of Theorem 1.1 is complete.

\section{Sampling in Bargmann-Fock spaces and concluding re- marks}

Finally, we give an application of Theorem 1.1 to several complex variables. Recall that the Bargmann-Fock space $\mathcal{F}$ consists of all entire functions on $\mathbb{C}^{d}$ with norm

$$
\|F\|_{\mathcal{F}}^{2}=\int_{\mathbb{C}^{d}}|F(z)|^{2} e^{-\pi|z|^{2}} d z .
$$

The Bargmann-Fock space possesses the reproducing kernel $K_{w}(z)=e^{\pi \bar{w} \cdot z}$ for $w, z \in \mathbb{C}^{d}$, so that $F(w)=\left(F, K_{w}\right)$. Consequently, a set $\left\{K_{\lambda}: \lambda \in \Lambda\right\}$ is a Riesz basis for $\mathcal{F}$ if and only if $\Lambda \subseteq \mathbb{C}^{d}$ is simultaneously sampling and interpolating for $\mathcal{F}$, i.e., $\sum_{\lambda}|F(\lambda)|^{2} e^{-\pi|\lambda|^{2}} \asymp\|F\|_{\mathcal{F}}^{2}$, and for every $c \in \ell^{2}(\Lambda)$ there exists a (unique) $F \in \mathcal{F}$, such that $F(\lambda) e^{-\pi|\lambda|^{2} / 2}=c_{\lambda}$.

The following result is an immediate consequence of Theorem 1.1:

Theorem 6.1. The Bargmann-Fock space does not admit a set $\Lambda \subseteq \mathbb{C}^{d}$ that is simultaneously sampling and interpolating.

Proof. We use the Bargmann transform defined as

$$
\mathcal{B} f(z)=F(z)=2^{d / 4} e^{-\pi z^{2} / 2} \int_{\mathbb{R}^{d}} f(t) e^{-\pi t \cdot t} e^{2 \pi t \cdot z} d t, \quad z \in \mathbb{C}^{d},
$$

and translate Theorem 1.1 into a statement of complex analysis. The Bargmann transform is unitary from $L^{2}\left(\mathbb{R}^{d}\right)$ onto $\mathcal{F}$ and maps the phase space shifts of the Gaussian $e^{-2 \pi i w_{2} \cdot x} e^{-\pi\left(x-w_{1}\right)^{2}}=\pi\left(w_{1},-w_{2}\right) g(x)$ to the reproducing kernel $e^{i \alpha} K_{w} e^{-|w|^{2} / 2}$ for some phase factor $|c|=1$. Thus $\Lambda \subseteq \mathbb{C}^{d}$ is a set of sampling and interpolation if and only if $\left\{K_{\lambda}: \lambda \in \Lambda\right\}$ is a Riesz basis for $\mathcal{F}$.

Clearly, the Gaussian satisfies the localization condition (5.1) for all $s>0$. By Theorem 1.1, a set of phase space shifts of the Gaussian cannot from a Riesz basis for $L^{2}\left(\mathbb{R}^{d}\right)$. Consequently, no set $\Lambda \subseteq \mathbb{C}^{d}$ can be simultaneously sampling and interpolating.

Remark. Theorem 6.1 is a statement of complex analysis. Indeed, in dimension $d=1$ is it well known and can be proved with classical methods from complex variables. In higher dimensions the result was expected, but seems to have been open so far. A proof with different methods has been announced in [1]. 
Final remarks. It is interesting to compare the critical value of the localization parameter $s$ in higher dimensions with the higher dimensional versions of the Balian-Low theorem. It is known that for $d=1$ and every $s<1$ there exists a function $f$ with

$$
\int_{\mathbb{R}}(1+|x|)^{2 s}|f|^{2}<\infty, \quad \int_{\mathbb{R}}(1+|\xi|)^{2 s}|\hat{f}|^{2}<\infty
$$

such that $\left\{e^{2 \pi i n t} f(x-m)\right\}_{n, m \in \mathbb{Z}}$ is an orthogonal basis for $L^{2}(\mathbb{R})$. (A more precise result is obtained in [4]; we refer the reader to [20] also.) Thus in the onedimensional setting the restrictions on the localization properties of an arbitrary orthogonal basis and of a Gabor system can be observed only at one point of our scale, $s=1$. The situation changes drastically when we consider higher dimensional spaces. There exists an orthogonal basis $\left\{f_{n}\right\}$ for $L^{2}\left(\mathbb{R}^{d}\right)$ that satisfies

$$
\sup _{n \in \mathbb{N}}\left(\inf _{a \in \mathbb{R}^{d}} \int_{\mathbb{R}^{d}}|x-a|^{2 d}\left|f_{n}(x)\right|^{2}+\inf _{b \in \mathbb{R}^{d}} \int_{\mathbb{R}^{d}}|\xi-b|^{2 d}\left|\widehat{f_{n}}(\xi)\right|^{2}\right)<\infty
$$

but for every orthogonal basis of the form $\left\{e^{2 \pi i n t} f(x-m)\right\}_{n, m \in \mathbb{Z}^{d}}$ a multidimensional version of Balian-Low theorem (see [9] and references therein) implies

$$
\sup _{n \in \mathbb{N}} \inf _{a \in \mathbb{R}^{d}} \int_{\mathbb{R}^{d}}|x-a|^{2}\left|f_{n}(x)\right|^{2}=\infty \quad \text { or } \quad \sup _{n \in \mathbb{N}} \inf _{b \in \mathbb{R}^{d}} \int_{\mathbb{R}^{d}}|\xi-b|^{2}\left|\widehat{f_{n}}(\xi)\right|^{2}=\infty .
$$

The reason for it could lie in the choice of the radial weight $v_{s}=|x|^{2 s}$. It seems that the product weight $w_{s}(x)=\left(\left(1+\left|x_{1}\right|\right) \cdots\left(1+\left|x_{d}\right|\right)\right)^{s}$ might be a more natural weight in higher dimensions.

There are a $(p, q)$-version of Bourgain's theorem [6] and a $(p, q)$ version of the Balian-Low theorem [13] for $1 / p+1 / q=1$. It would be interesting to obtain a $(p, q)$-version of Theorem 1.1 .

Acknowledgment. K. G. would like to thank the Department of Mathematics of NTNU Trondheim for its hospitality and the great research environment during the work on this paper. Both authors would like to thank the Centre de Recerca Matemàtica Barcelona for ideal conditions for completing this work.

\section{References}

[1] Ascensi, G., Feichtinger, H. G. and Kaiblinger, N.: Dilation of the Weyl symbol and Balian-Low theorem. To appear in Trans. Amer. Math. Soc.

[2] Balan, R., Casazza, P. G., Heil, C. and Landau, Z.: Density, overcompleteness, and localization of frames. I. Theory. J. Fourier Anal. Appl. 12 (2006), no. 2, 105-143.

[3] Battle, G.: Heisenberg proof of the Balian-Low theorem. Lett. Math. Phys. 15 (1988), no. 2, 175-177.

[4] Benedetto, J., Czaja, W., Gadziński, P. And Powell, A.: The Balian-Low theorem and regularity of Gabor systems. J. Geom. Anal. 13 (2003), no. 2, 239-254. 
[5] Benedetto, J., Heil, C. And Walnut, D.: Differentiation and the Balian-Low theorem. J. Fourier Anal. Appl. 1 (1995), no. 4, 355-402

[6] Benedetto, J. And Powell, A.: A $(p, q)$ version of Bourgain's theorem. Trans. Amer. Math. Soc. 358 (2006), no. 6, 2489-2505.

[7] Bourgain, J.: A remark on the uncertainty principle for Hilbertian basis. J. Funct. Anal. 79 (1988), no. 1, 136-143.

[8] Cordero, E. And Gröchenig, K.: Time-frequency analysis of localization operators. J. Funct. Anal. 205 (2003), no. 1, 107-131.

[9] Czaja W. And Powell, A.: Recent developments in the Balian-Low theorem. In Harmonic analysis and applications, 79-100. Appl. Numer. Harmon. Anal., Birkhäuser, Boston, MA, 2006.

[10] Daubechies, I.: The wavelet transform, time-frequency localization and signal analysis. IEEE Trans. Inform. Theory 36 (1990), no. 5, 961-1005.

[11] Folland, G.B. And Sittaram, A.: The uncertainty principle: a mathematical survey. J. Fourier Anal. Appl. 3 (1997), no. 3, 207-238.

[12] Fornasier, M. And Gröchenig, K.: Intrinsic localization of frames. Constr. Approx. 22 (2005), no. 3, 395-415.

[13] Gautam, S.Z.: A critical-exponent Balian-Low theorem. Math. Res. Lett. 15 (2008), no. 3, 471-483.

[14] Gröchenig, K.: Foundations of time-frequency analysis. Applied and Numerical Harmonic Analysis. Birkhäuser, Boston, MA, 2001.

[15] Gröchenig, K.: Localization of frames, Banach frames and the invertibility of the frame operator. J. Fourier Anal. Appl. 10 (2004), no. 2, 105-132.

[16] Gröchenig, K. and Razafinjatovo, H.: On Landau's necessary density conditions for sampling and interpolation of band-limited functions. J. London Math. Soc. (2) $\mathbf{5 4}$ (1996), no. 3, 557-565.

[17] HeIl, C.: History and evolution of the density theorem for Gabor frames. J. Fourier Anal. Appl. 13 (2007), no. 2, 113-166.

[18] Jaffard, S.: Propriétés des matrices "bien localisées" près de leur diagonale et quelques applications. Ann. Inst. H. Poincaré Anal. Non Linéaire 7 (1990), no. $5,461-476$.

[19] Jaming, P. And Powell, A. M.: Time-frequency concentration of generating systems. Proc. Amer. Math. Soc. 139 (2011), no. 9, 3279-3290.

[20] Janssen, A.J.E. M.: A decay result for certain windows generating orthogonal Gabor bases. J. Fourier Anal. Appl. 14 (2008), no. 1, 1-15.

[21] Landau, H. J. and Pollak, H. O.: Prolate spheroidal wave functions, Fourier analysis and uncertainty. III. The dimension of the space of essentially time- and band-limited signals. Bell System Tech. J. 41 (1962), 1295-1336.

[22] Landau, H. J.: Necessary density conditions for sampling and interpolation of certain entire functions. Acta Math. 117 (1967), 37-52.

[23] Ramanathan, J. and Steger, T.: Incompleteness of sparse coherent states. Appl. Comput. Harmon. Anal. 2 (1995), no. 2, 148-153.

[24] Slepian, D. And Pollak, H. O.: Prolate spheroidal wave functions, Fourier analysis and uncertainty. I. Bell System Tech. J. 40 (1961), 43-64. 
[25] Slepian, D.: Prolate spheroidal wave functions, Fourier analysis and uncertainity. IV. Extensions to many dimensions; generalized prolate spheroidal functions. Bell System Tech. J. 43 (1964), 3009-3057.

[26] Wong, M. W.: Localization operators. Lecture Notes Series 47, Seoul National University Research Institute of Mathematics, Global Analysis Research Center, Seoul, 1999.

Received February 16, 2011.

KARLheinz Gröchenig: Faculty of Mathematics, University of Vienna, Nordbergstrasse 15, A-1090 Vienna, Austria.

E-mail: karlheinz.groechenig@univie.ac.at

Eugenia Malinnikova: Department of Mathematical Sciences, Norwegian University of Science and Technology, 7491, Trondheim, Norway.

E-mail: eugenia@math.ntnu.no

K. G. was supported in part by the project P22746-N13 of the Austrian Science Foundation (FWF). E. M. was supported by the Research Council of Norway grant 185359/V30. 